\title{
THE SPHERICAL BERNSTEIN PROBLEM IN EVEN DIMENSIONS
}

\author{
BY PER TOMTER
}

Introduction. The minimal surface equation is probably the best known nonlinear elliptic partial differential equation, and it has been studied extensively. After the settling of the classical Bernstein conjecture through the combined efforts of de Giorgi, Almgren, Simons, Bombieri and Giusti (19651969), S. S. Chern, at the International Congress of Mathematicians in Nice, 1970, proposed the spherical Bernstein problem [3]:

Let the $(n-1)$-sphere be imbedded as a minimal hypersurface in the standard sphere $S^{n}$. Is it necessarily an equator?

The problem is of clear significance in differential geometry, being closely related to the study of minimal cones and the local topological structure of isolated singularities in minimal hypersurfaces of riemannian manifolds.

For $n=3$ the answer is positive by a theorem of Almgren and Calabi, which holds under the weaker assumption of an immersed $S^{2}$. No progress was made on the problem until Wu-Yi Hsiang recently proved the existence of infinitely many noncongruent minimal imbeddings of $S^{n-1}$ into $S^{n}$ for the specific dimensions $n=4,5,6,7,8,10,12,14[4,5]$.

It is the purpose of this note to announce the solution of the spherical Bernstein problem for all even $n$. There are notable differences between the examples for $S^{2 m}, m \geq 8$, constructed here, and those referred to above. We are indebted to Professor Wu-Yi Hsiang for introducing us to his work in equivariant differential geometry and for his encouragement.

\section{Main result.}

THEOREM. Let $S^{2 m}$ be the standard sphere of dimension $2 m$. Then there exists a minimally imbedded (2m-1)-sphere which is different from the equator.

We believe there exists only one congruence class of such examples which are invariant and of cohomogeneity one under the isometry subgroup $\mathrm{SO}(2) \times$ $\mathrm{SO}(m)$ on $S^{2 m}, m \geq 8$, described below. The existence of such examples is reduced to the existence of certain types of solution curves of a second order differential equation in the two-dimensional orbit space of an isometry group. In Hsiang's proof it is essential that the singularity of an auxiliary two-dimensional dynamical system (which locally approximates the corner singularity of the orbit space) is of focal type. For cohomogeneity two isometry subgroups of spheres in all other dimensions, however, this singularity is a nodal point. It requires a considerable amount of analysis in orbit space to deal with that case.

Received by the editors January 3, 1984.

1980 Mathematics Subject Classification. Primary 53C42, 53A70.

(c) 1984 American Mathematical Society $0273-0979 / 84 \$ 1.00+\$ .25$ per page 
The same type of construction gives a $\mathrm{S}(\mathrm{U}(2) \times \mathrm{U}(m)$ )-invariant example on $S^{4 m}$ and a $\mathrm{Sp}(2) \times \mathrm{Sp}(m)$-invariant example on $S^{8 m}$.

OUtline OF THE Construction. The construction is based on the orbital geometry of the group $G=\mathrm{SO}(2) \times \mathrm{SO}(m)$ acting on $S^{2 m} \subseteq \mathbf{R}^{2 m+1}=$ $\mathbf{R}^{2} \otimes \mathbf{R}^{m} \oplus \mathbf{R}$ by the representation $\rho_{2} \otimes \rho_{m} \oplus 1$.

1. The orbit space $S=S^{2 m} / G$ is a spherical lune, parameterized in spherical polar coordinates by $(r, \theta) \in[0, \pi] \times[0, \pi / 4]$.

2. A curve in $X$ is the projection of a minimal hypersurface in $S^{2 m}$ iff it satisfies the differential equation:

$$
\begin{aligned}
& \frac{d r}{d s}=\cos \alpha, \quad \frac{d \theta}{d s}=\frac{\sin \alpha}{\sin r}, \\
& \frac{d \alpha}{d s}=-(2 m-1) \frac{\sin \alpha}{\sin r} \cos r+2 \frac{\cos \alpha}{\sin r}((m-2) \cot 2 \theta-\tan 2 \theta),
\end{aligned}
$$

where the curve is parameterized by arc-length $s$, and $\alpha$ is the angle from $\partial / \partial r$ to the tangent vector of the curve.

3. Any curve in $X$ which starts orthogonally to the boundary of $X$ at a point $(r, \theta)=(b, 0)$ and ends orthogonally to the boundary at a point $(r, \theta)=$ $(c, \pi / 4), b, c \in(0, \pi)$, is the projection to $X$ of a smooth hypersphere in $S^{2 m}$.

4. Let $b \in(0, \pi)$. There is a unique solution curve $\gamma_{b}(s)=(r(s), \theta(s), \alpha(s))$ of $(*)$ with $r(0)=b, \theta(0)=0$ (or $\pi / 4$ ), and $d \theta(0) / d s>0$. For this curve $\alpha(0)=\pi / 2$.

5. Let $I(b)$ be the number of intersection points of $\gamma_{b}(s)$ with solution curve $\theta \equiv \theta_{0}=\frac{1}{2}$ Arctan $\sqrt{m-2}$ before $r(s)$ reaches its first maximum.

LEMMA 1. There is an $\epsilon>0$ such that $I(b) \leq 2$ for $b \in(\pi / 2-\epsilon, \pi / 2)$.

LEMMA 2. There is an $\epsilon>0$ such that $I(b) \geq 4$ for $b \in(0, \epsilon)$.

By studying the variation of $I(b)$ along the one-parameter family of solution curves $\left\{\gamma_{b}\right\}$, it is now easy to conclude from the lemmas that for some value of $b, \gamma_{b}$ must intersect the boundary $\theta \equiv \pi / 4$ before any self-intersection. This is sufficient to establish our main theorem.

The proof of the lemmas requires rather explicit estimates as well as qualitative arguments. Lemma 2 is particularly delicate. The intersection pattern with the solution $\theta \equiv \theta_{0}$ is mainly controlled by estimating the auxiliary function

$$
v=\frac{\alpha}{\theta-\theta_{0}}+\frac{2 m-1}{2} \cos r
$$

along the orbit. The limit differential equation satisfied by $v$ when $\alpha \rightarrow 0$, $\theta \rightarrow \theta_{0}$ is

$$
\frac{d v}{d s}=\frac{1}{\sin r}\left[-v^{2}+\frac{4 m^{2}-1}{4} \cos ^{2} r-\frac{18 m-17}{2}\right] .
$$

The intersections take place in the region

$$
\cos ^{2} r<2 \frac{18 m-17}{4 m^{2}-1} \text {. }
$$

The proof of the main theorem and further discussion of the spherical Bernstein problem will be published elsewhere. 


\section{REFERENCES}

1. F. J. Almgren, Jr., Some interior regularity theorems for minimal surfaces and an extension of Bernstein's theorem, Ann. of Math. (2) 85 (1966), 277-292.

2. E. Bombieri, E. Giorgi and E. Giusti, Minimal cones and the Bernstein problem, Invent. Math. 7 (1969), 243- 268.

3. S. S. Chern, Differential geometry, its past and future, Actes Congrès Internat. Math. (Nice, 1970), Tome 1, pp. 41-53.

4. W. Y. Hsiang, Minimal cones and the spherical Bernstein problem. I, Ann. of Math. (2) 118 (1983), 61-75.

5. __ Minimal cones and the spherical Bernstein problem. II, Invent. Math. 74 (1983), 351-369.

6. J. Simons, Minimal varieties in riemannian manifolds, Ann. of Math. (2) 88 (1968), 62-105.

Institute of Mathematics, University of Oslo, Blindern, Oslo 3, Norway 